\title{
CUGC for Simpson-Golabi-Behmel syndrome (SGBS)
}

\author{
Marie-Laure Vuillaume $\mathbb{1}^{1,2} \cdot$ Marie-Pierre Moizard ${ }^{1,2} \cdot$ Alessandra Baumer $^{3} \cdot$ Edouard Cottereau $^{1} \cdot$ \\ Frédéric Brioude $\mathbb{C}^{4,5} \cdot$ Anita Rauch $\mathbb{D}^{3} \cdot$ Annick Toutain $\mathbb{1}^{1,2}$
}

Received: 12 September 2018 / Revised: 14 December 2018 / Accepted: 25 December 2018 / Published online: 25 January 2019

(c) European Society of Human Genetics 2019

\section{Disease characteristics}

\subsection{Name of the disease (Synonyms)}

Simpson-Golabi-Behmel syndrome (SGBS).

\subsection{OMIM\# of the disease}

312870.

\subsection{Name of the analysed genes or DNA/ chromosome segments}

GPC3.

\subsection{OMIM\# of the gene(s)}

$300037 .{ }^{1}$

Annick Toutain

annick.toutain@univ-tours.fr

1 Service de Génétique, Centre Hospitalier Universitaire, Tours, France

2 UMR 1253, iBrain, Université de Tours, INSERM, Tours, France

3 Institute of Medical Genetics, University of Zurich, SchlierenZurich, Switzerland

4 Explorations Fonctionnelles Endocriniennes, APHP, Hôpital Trousseau, Paris, France

5 Sorbonne Université, INSERM UMR_S938 Centre de Recherche Saint-Antoine, AP-HP Hôpital Trousseau, Paris, France

\footnotetext{
1 This OMIM number corresponds to SGBS type 1. However, we consider that Simpson-Golabi-Behmel syndrome type 2 does not exist, as it has been demonstrated that this designation refers to a totally different condition caused by variants in the PIGA gene. There is therefore only one Simpson-Golabi-Behmel syndrome and it is no longer justified to speak of SGBS type 1 .
}

\subsection{Mutational spectrum}

Simpson-Golabi-Behmel syndrome (SGBS) is an X-linked multiple congenital anomalies (MCA) and overgrowth syndrome characterized in males by foetal macrosomia, postnatal overgrowth, macrocephaly, organomegaly, particular facial features, extremities abnormalities, supernumerary nipples, cardiac, skeletal, gastrointestinal and genitourinary malformations, tumour predisposition, especially Wilms and liver tumours, and a variable degree of learning disabilities in some cases [1,2]. Female carriers are usually asymptomatic, but some are noted to have mild features detected following detailed clinical assessment after diagnosis in a male relative. Therefore, female carriers are usually not index cases although it has been reported twice in the literature [3]. SGBS is caused by a loss-offunction of the glypican-3 gene (GPC3). This gene located at Xq26.3 has a full-length transcript of $2.3 \mathrm{~kb}$ (NCBI reference sequence NM_004484.3) and contains 8 exons. In 2011, GPC4, a gene coding for another member of the glypican family, and located close to GPC3 at its 3 '-end, was also suggested to be associated with Simpson-GolabiBehmel syndrome. However, only one duplication of all 9 GPC4 exons, of which the functional consequences have not been assessed, has been reported in the original family described by Golabi and Rosen [4]. Moreover, no GPC4 point variants have been reported so far, questioning the exact role of this gene in the pathogenesis of SGBS [5].

Up to now, GPC3 remains the only gene surely involved in SGBS. To date, 86 distinct GPC3 variants have been reported in 120-unrelated patients [6]. These variants, mostly inherited, are dispersed throughout the whole-coding region of the gene with no obvious variant hotspots. The majority of these variants lead to the occurrence of a premature stop codon. Although all types of variant are found, the most prevalent type is large deletions $(34.9 \%)$ followed by frameshift variants $(24.4 \%)$, nonsense variants $(16.3 \%)$, missense variants $(8.1 \%)$, large duplications $(8.1 \%)$, splice site variants $(4.7 \%)$, translocations $(2.3 \%)$ and one in frame 
insertion/deletion leading to a stop codon (1.2\%). Many of these variants are listed in the Leiden Open Variation Database (LOVD) (https://databases.lovd.nl/shared/varia nts/GPC3).

\subsection{Analytical methods}

Initial diagnosis of SGBS is usually considered clinically on suggestive features and, in some cases, family history consistent with X-linked inheritance. A combined approach based on PCR, direct sequencing and MLPA analysis is then conducted on genomic DNA extracted from peripheral blood in order to identify GPC3 variants and to confirm the clinical diagnosis of SGBS.

In affected males, as exon deletions are the most prevalent type of variant, small intragenic GPC3 deletions are firstly screened by PCR amplification. In the absence of intragenic GPC3 deletion, bidirectional sequencing (Sanger) of all coding exons and of the exon-intron boundaries is carried out. In case of splice site variants, a GPC3 transcript molecular analysis can be conducted by Reverse Transcription (RT)-PCR amplification with cDNA primers flanking the potentially skipped exon, and subsequent Sanger sequencing of the cDNA fragment. If no point variant is identified, a search for a GPC3 intragenic duplication or a mosaic deletion is usually performed by Multiplex Ligation-dependent Probe Amplification (MLPA) analysis. In female probands, MLPA would be the first diagnostic step, followed by sequencing.

In most cases, variants are identified by a targeted analysis of GPC3 in patients clinically diagnosed with SGBS. However, with the advent of high-throughput technologies, new GPC3 rare variants are also revealed incidentally either by Array Comparative Genomic Hybridization (Array$\mathrm{CGH}$ ), targeting gene sequencing panels or whole exome/ genome sequencing. Currently, GPC3 is present in different targeting gene sequencing panels such as overgrowth and intellectual disability panels.

\subsection{Analytical validation}

The variant is confirmed by sequencing a second independently amplified PCR product from the patient's DNA. In case of non-amplification of a single amplicon, MLPA should be performed to confirm a deletion and exclude allelic drop out. In case of whole GPC3 rearrangement or when the $5^{\prime}$ - or $3^{\prime}$-end of GPC3 is involved in the rearrangement, chromosomal microarray can be used in order to fine-map the rearrangement breakpoints. Segregation analysis of variants of unknown significance can be performed in the family by testing male relatives, in order to determine their pathogenicity.

\subsection{Estimated frequency of the disease}

(Incidence at birth ("birth prevalence") or population prevalence. If known to be variable between ethnic groups, please report).

SGBS is very rare and its exact prevalence is unknown. In a recent review of the literature we have identified 152 male patients, belonging to 120 -unrelated families, with a GPC3 variant [6]. In their review, Tenorio et al. [7] mentioned that 250 patients had been reported in the literature, but they included patients for whom misdiagnosis was possible as no confirmation by a molecular analysis was performed. On the other hand, one may suspect that SGBS may still be underdiagnosed due to unfamiliarity with the phenotype amongst physicians.

\subsection{Diagnostic setting}

\begin{tabular}{lcc}
\hline & Yes. & No. \\
\hline A. (Differential) diagnostics & $\bigotimes$ & $\square$ \\
B. Predictive testing & $\square$ & $\square$ \\
C. Risk assessment in relatives & $\bigotimes$ & $\square$ \\
D. Prenatal & $\square$ & $\square$ \\
\hline
\end{tabular}

Comment: SGBS is an X-linked disorder with full expression in male patients. Female carriers are usually asymptomatic, although there is increasing evidence that some carrier females may have mild features (unpublished data of the authors), but only seven reports are found in the literature until now [3].

The main differential diagnosis of SGBS is BeckwithWiedemann syndrome (BWS) which, besides overgrowth, shares many clinical features, such as macrosomia, macroglossia and visceromegaly, coarse facial features with grooves on the ear lobes, hypoglycaemia, and the same spectrum of embryonic tumours. These two syndromes are nevertheless distinguishable as BWS patients have a normal $\mathrm{OFC}$ and rarely have congenital malformations, apart from omphalocele, which is not part of the SGBS phenotype. A phenotypic overlap also exists with other overgrowth syndromes such as Sotos, Weaver and Perlman syndromes but is less striking. Physicians experienced in the clinical diagnosis of overgrowth syndromes may easily distinguish between these conditions for patients with a classical presentation, but some patients may have mild or atypical presentations. A molecular overlap has also been reported between SGBS and BWS, as some patients with an initial clinical diagnosis of BWS may have variants in $G P C 3$, and patients with an initial clinical diagnosis of SGBS may have 
molecular anomalies within the $11 \mathrm{p} 15$ region (unpublished data of the authors).

As penetrance is complete in male patients and since the disease is present from birth, molecular diagnosis is not used for predictive testing. A careful clinical assessment is however recommended in at-risk male relatives.

A molecular diagnosis is possible in relatives of SGBS individuals to identify female carriers and to screen other atrisk male relatives.

Prenatal testing for SGBS is also possible for at-risk pregnancies when a variant has been identified in the family. Molecular genetic testing can also be conducted when prenatal ultrasound examination detect foetal overgrowth associated with polyhydramnios and often elevated maternal serum alpha-fetoprotein (AFP) levels.

\section{Test characteristics}

$\begin{array}{ll}\begin{array}{l}\text { Genotype or A: true positives } \\ \text { disease }\end{array} & \text { C: false } \\ \text { negative } \\ \text { Present Absent B: false positives } & \text { D: true } \\ & \text { negative }\end{array}$

Test

\begin{tabular}{llll} 
Pos. A & B & Sensitivity:Specificity: & A/(A+C) \\
& & & D/(D+B) \\
Neg. C & D & $\begin{array}{l}\text { Pos. predict. value: } \\
\text { Neg. predict. value: }\end{array}$ & A/(A+B) \\
& & D/(C+D) \\
\hline
\end{tabular}

\subsection{Analytical sensitivity}

(Proportion of positive tests if the genotype is present).

Nearly $100 \%$ when the variant is located within the coding exons and flanking introns of GPC3. Indeed, in routine analysis, only these regions are screened in SGBS patients using Sanger sequencing. Deep intronic variants or other variants located within the $5^{\prime}$ - or $3^{\prime}$-UTRs of the gene and disrupting regulatory regions cannot be detected, but are expected to be extremely rare (such variants have not yet been described even with the advent of Next Generation Sequencing).

\subsection{Analytical specificity}

(Proportion of negative tests if the genotype is not present). Nearly $100 \%$.

\subsection{Clinical sensitivity}

(Proportion of positive tests if the disease is present).

The clinical sensitivity can be dependent on variable factors, such as age or family history. In such cases a general statement should be given, even if a quantification can only be made case by case.

The variant detection rate in SGBS is highly variable depending on the reports [5]. Low detection rates may reflect less stringent clinical criteria used by the laboratories and/or clinical misdiagnosis due to the unfamiliarity with the phenotype amongst the clinicians requesting GPC3 analysis.

\subsection{Clinical specificity}

(Proportion of negative tests if the disease is not present).

The clinical specificity can be dependent on variable factors, such as age or family history. In such cases a general statement should be given, even if a quantification can only be made case by case.

Nearly $100 \%$.

\subsection{Positive clinical predictive value}

(Life time risk to develop the disease if the test is positive). $100 \%$ in males as SGBS is an X-linked disorder and GPC3 variants are fully penetrant in males.

\subsection{Negative clinical predictive value}

(Probability not to develop the disease if the test is negative).

Assume an increased risk based on family history for a non-affected person. Allelic and locus heterogeneity may need to be considered.

Index case in that family had been tested:

$100 \%$.

Index case in that family had not been tested:

Nearly $100 \%$ (see 2.1 and 2.5), but genetic testing would usually not be performed in asymptomatic male individuals.

\section{Clinical utility}

\section{1 (Differential) diagnostics: the tested person is clinically affected}

(To be answered if in 1.9 "A" was marked). 


\subsubsection{Can a diagnosis be made other than through a genetic test?}

\begin{tabular}{lll}
\hline No & $\square$ (continue with 3.1.4) & \\
Yes & $\bigotimes$ & $\square$ \\
& Clinically & $\square$ \\
Imaging & $\square$ \\
Endoscopy & $\square$ \\
Biochemistry & $\square$ \\
& Electrophysiology & \\
& Other (please describe)
\end{tabular}

\subsubsection{Describe the burden of alternative diagnostic methods to the patient}

SGBS usually has a recognizable clinical picture and should be strongly suspected clinically in the majority of cases. However, in cases with a clinical doubt, where other overgrowth syndromes may be hypothesized (typically a presumed Beckwith-Wiedemann case with no 11p15 molecular defect), imaging techniques are often performed in order to detect internal malformations or skeletal problems which may bring further arguments for the diagnosis. The molecular proof of a GPC 3 variant precludes further investigations for overgrowth. These investigations are nevertheless important for the management of the patients. Moreover, molecular genetic testing is still essential to confirm the diagnosis allowing reliable genetic counselling and prenatal diagnosis if desired.

\subsubsection{How is the cost-effectiveness of alternative diagnostic methods to be judged?}

Not applicable (see 3.1.2).

\subsubsection{Will disease management be influenced by the result of a genetic test?}

\section{No}

Yes $\otimes$

Therapy (please describe)
Treatment is mostly symptomatic, including, when necessary, treatment of neonatal hypoglycemia, surgery for congenital malformations or macroglossia, tumour management, treatment of arrhythmia.
Table (continued)

Prognosis (please Prognosis is relatively good in the describe) majority of cases but depends on the range of clinical features. In some cases, SGBS may be lifethreatening at birth or in infancy due to congenital malformations, mostly diaphragmatic hernia. In the other cases, patients usually have a normal life expectancy, although cardiac complications and tumours may also be a cause of premature death, but the multiplicity of required medical interventions may be a burden in daily life. Moderate/ mild psychomotor delay and/or learning difficulties and behavioural problems may be a cause of handicap and lack of autonomy in adulthood.

Management Management of SGBS requires a (please describe) multidisciplinary team with paediatric specialists (cardiologists, neurologists, orthopaedists, surgeons, audiologists, otorhinolaryngologists, ophthalmologists, oncologists), physiotherapists and speech therapists. As in other MCA syndromes, hearing evaluation, eye examination, screening for internal malformations and skeletal problems (e.g. scoliosis) in order to detect problems, which may require medical intervention are necessary in every patient diagnosed with SGBS. Neurodevelopmental assessment may be necessary to determine the need for special education or occupational therapy.

In SGBS a particular attention is required on cardiac complications and tumour risk.

In addition to the detection of heart defects at the time of the diagnosis, a regular cardiac follow-up is necessary in order to detect and treat heart conduction and rhythm problems. 
Table (continued)

Specific screening for tumours especially Wilms tumours, liver tumours and gonadoblastoma, is recommended in both affected males and symptomatic carrier females, although there is no international consensus on that subject at the moment. Awaiting for international guidelines, one may recommend, as in other overgrowth syndromes with increased tumour risk, repeated abdominal ultrasound scans and alphafoetoprotein measurements.
3.2 Predictive setting: the tested person is clinically unaffected but carries an increased risk based on family history

(To be answered if in 1.9 "B" was marked).

\subsubsection{Will the result of a genetic test influence lifestyle and prevention?}

If the test result is positive (please describe) Not applicable. If the test result is negative (please describe) Not applicable.

3.2.2 Which options in view of lifestyle and prevention does a person at-risk have if no genetic test has been done (please describe)?

Not applicable.

\subsection{Genetic risk assessment in family members of a diseased person}

(To be answered if in 1.9 "C" was marked).

\subsubsection{Does the result of a genetic test resolve the genetic situation in that family?}

Yes. It confirms X-linked inheritance and is the prerequisite for genetic risk assessment in female relatives and prenatal diagnosis.

3.3.02 Can a genetic test in the index patient save genetic or other tests in family members?

Yes. Genetic testing is not absolutely necessary in a male relative of an index case with a positive genetic test, when he has definitive clinical symptoms. The proof of a de novo occurrence of the variant in an index case can save genetic tests in other family members. However, germline mosaicism, even if rare, should be kept in mind as it has already been described in one family [8].

3.3.3 Does a positive genetic test result in the index patient enable a predictive test in a family member?

No see comments 1.9B. The test is only performed to identify female carriers. Testing is not performed in nonaffected males. However, as some male patients may present only mild or unique symptoms (e.g. overgrowth, renal problems), genetic testing may be used in male relatives in case there is a clinical doubt.

\subsection{Prenatal diagnosis}

(To be answered if in 1.9 "D" was marked).

3.4.1 Does a positive genetic test result in the index patient enable a prenatal diagnosis?

Yes. Molecular prenatal diagnosis can then be offered to the female carriers of the GPC3 variant.

\section{If applicable, further consequences of testing}

Please assume that the result of a genetic test has no immediate medical consequences. Is there any evidence that a genetic test is nevertheless useful for the patient or his/her relatives? (Please describe).

A genetic diagnosis often has a positive psychological impact on the parents of an affected child, as it provides a definitive explanation for the cause of the disease. Identification of the causative variant also avoids serial misdiagnosis and further unnecessary investigations, and allows more appropriate management and follow-up of the index patient and of male relatives carrying the familial variant. Moreover, this diagnosis impacts reproductive decision of female carriers and allows appropriate genetic counselling.

Acknowledgements This work was supported by the EuroGentest2 (Unit 2: "Genetic testing as part of health care"), a Coordination Action under FP7 (Grant Agreement Number 261469) and the European Society of Human Genetics.

\section{Compliance with ethical standards}

Conflict of interest The authors declare that they have no conflict of interest. 
Publisher's note: Springer Nature remains neutral with regard to jurisdictional claims in published maps and institutional affiliations.

\section{References}

1. Golabi M, Leung A, Lopez C. Simpson-Golabi-Behmel syndrome type 1. In: Pagon RA, Adam MP, Ardinger HH, Wallace SE, Amemiya A, Bean LJ, et al., editors. GeneReviews $\left.{ }^{\circledR}\right)$ [Internet]. Seattle (WA): University of Washington; 2011.

2. Cottereau E, Mortemousque I, Moizard M-P, Bürglen L, Lacombe D, Gilbert-Dussardier B, et al. Phenotypic spectrum of SimpsonGolabi-Behmel syndrome in a series of 42 cases with a mutation in GPC3 and review of the literature. Am J Med Genet C Semin Med Genet. 2013;163C:92-105.

3. Schirwani S, Novelli A, Digilio MC, Bourn D, Wilson V, Roberts $\mathrm{C}$, et al. Duplications of GPC3 and GPC4 genes in symptomatic female carriers of Simpson-Golabi-Behmel syndrome type 1. Eur J Med Genet. 2018. (In press).
4. Waterson J, Stockley TL, Segal S, Golabi M. Novel duplication in glypican-4 as an apparent cause of Simpson-Golabi-Behmel syndrome. Am J Med Genet A. 2010;152A:3179-81.

5. Cottereau E, Moizard M-P, David A, Raynaud M, Marmin N, Toutain A. Duplication of exon 2 of the GPC3 gene in a case of Simpson-Golabi-Behmel syndrome. Am J Med Genet A. 2014;164A:282-4.

6. Vuillaume M-L, Moizard M-P, Rossignol S, Cottereau E, Vonwill $\mathrm{S}$, Alessandri JL, et al. Mutation update for the GPC3 gene involved in Simpson-Golabi-Behmel syndrome and review of the literature. Hum Mutat. 2018;39:790-805.

7. Tenorio J, Arias P, Martínez-Glez V, Santos F, García-Miñaur S, Nevado J, et al. Simpson-Golabi-Behmel syndrome types I and II. Orphanet J Rare Dis. 2014;9:138.

8. Agatep R, Shuman C, Steele L, Parkinson N, Weksberg R, Stockley TL. Paternal germline mosaicism for a GPC3 deletion in X-linked Simpson-Golabi-Behmel syndrome. Am J Med Genet A. 2014;164A:2682-4. 